\title{
Numerical identification of blade exit angle effect on the performance for a multistage centrifugal pump impeller
}

\author{
Osman Babayigit ${ }^{1, a}$, Osman Kocaaslan ${ }^{2}$, Muharrem Hilmi Aksoy ${ }^{3}$, Kursad Melih Guleren $^{4}$ and Muammer Ozgoren ${ }^{3}$ \\ ${ }^{1}$ Selcuk University, Hadim Vocational School, 42830 Konya, Turkey \\ ${ }^{2}$ Selcuk University, Huglu Vocational School, 42710 Konya, Turkey \\ ${ }^{3}$ Selcuk University, Engineering Faculty, Mechanical Engineering Department, 42100 Konya, Turkey. \\ ${ }^{4}$ University of Turkish Aeronautical Association, Ankara, Turkey
}

\begin{abstract}
Nowadays, single and multistage centrifugal pumps are widely used in industrial and mining enterprises. One of the most important components of a centrifugal pump is the impeller. The performance characteristics are related to the pump comprising the head and the overall efficiency rely a great deal on the impeller geometry. In this work, effects of blade exit angle change on hydraulic efficiency of a multi stage pump impeller are investigated via Ansys-Fluent computational fluid dynamics software for constant width impeller entrance and exit gates, blade numbers and blade thickness. Firstly, the flow volume of a centrifugal pump impeller is generated and then mesh structure is formed for the full impeller flow volume. Secondly, rotational periodic flow model are adopted in order to examine the effect of periodic flow assumption on the performance predictions. Corresponding to the available experimental data, inlet mass flow rate, outlet static pressure and rotation of impeller are taken as $0.02 \mathrm{~m}^{3} \mathrm{~s}^{-1}, 450 \mathrm{kPa}$ and $2950 \mathrm{rpm}$, respectively for the water fluid. No slip boundary condition is exposed to all solid of surface in the flow volume. The continuity and Navier-Stokes equations with the $k-\varepsilon$ turbulence model and the standard wall functions are used. During the study, numerical analyses are conducted for the blade exit angle values of $18^{\circ}, 20^{\circ}, 25^{\circ}$, $30^{\circ}$ and $35^{\circ}$. In consequence of the performed analyses, it is determined that hydraulic efficiency of the pump impeller value is changed between $81.0-84.6 \%$. The most convenient blade exit angle that yields $84.6 \%$ hydraulic efficiency at is $18^{\circ}$. The obtained results show that the blade exit angle range has an impact on the centrifugal pump performance describing the pump head and the hydraulic efficiency.
\end{abstract}

Keywords: Blade exit angle, centrifugal pump, CFD, hydraulic efficiency, impeller, k- $\varepsilon$ turbulence model, periodic analysis.

\section{Introduction}

Electrical energy is converted into mechanical energy through pumps that are well known class of turbomachinery. Pump systems are widely used in water supply, industrial machinery, water transferring systems, agricultural irrigation, water conditioning, heating, cooling and ventilation systems. Considering life-cycle cost, improvements that can be achieved in the efficiency of the pumps has a great importance $[1,2]$.

A large number of the pumping systems are still designed and produced by conventional methods, and because of that they run at low efficiency. In order to increase pump efficiency, detailed investigation about the flow in impeller is still required. Studies regarding the elaborate investigation of the flow in pumps can provide the determination of unwanted physical attributes of the flow and the elimination of them with smart engineering solutions.

\footnotetext{
${ }^{\mathrm{a}}$ Corresponding author: obabayigit@selcuk.edu.tr
}

The impeller is the most important part in a centrifugal pump since it is the place where the mechanical energy is converted into hydraulic energy. Hence the parameters related to the impeller are directly affecting the performance of the pump [3]. One of them is the blade exit angle; if it is not correctly designed, it can negatively affect the head and the hydraulic efficiency of the pump.

There are studies about the effect of the blade angles and geometries on hydraulic efficiency. Some of them are impeller exit angles [4-7], blade wrap angle [8,9], blade thickness [10] and impeller exit width [11]. Anagnostopoulos [12] investigated the effect of blade geometry on pump efficiency using his 2-D turbulent flow solver. It is emphasized that flow rate decreases at low blade angles and increases at high blade angles with increasing the losses. Optimum blade angle can be found after an elaborate analysis. Kypasarissiset et al. [13] showed that double curvature blades are more suitable for high efficiency pumps. Sanda and Daniela [14] expressed 
that variation of blade inlet angles had an effect of 1-2\% on hydraulic efficiency, which might be very important when considered the total energy consumption of the pump in the world.

Bayraktar and Eralp [15] used a computer-aided design method based on experimental techniques for centrifugal pump design in their study. They obtained 3$\mathrm{D}$ model of the design with data transmission to CAD/CAM programs. They confirmed validation of their developed program according to the pump design method in the literature. Their results showed that the method is reliable. Liu et al. [16] investigated numerically with a simulation of inner-flow in a multi-stage pump. They used Fluent software and standard k- $\varepsilon$ model for this study. They used three different types of impeller for the numerical analysis which are twisted type, cylindrical type and stopper type.

In the present study, effects of blade exit angle change on hydraulic efficiency of a multi stage pump impeller are investigated via Ansys-Fluent computational fluid dynamics software for constant width impeller entrance and exit gates, blade numbers and blade thickness. Firstly, the flow volume of a centrifugal pump impeller is generated and then mesh structure is formed for the full impeller flow volume. Secondly, rotational periodic flow model is adopted in order to examine the effects of periodic flow assumption on the performance predictions.

\section{Material and methods}

In this study, a multi stage centrifugal impeller with a vertical shaft pump is selected. Numerical analyses of the flow in the pump are performed considering both the full impeller and periodic flow volume in order to understand whether the periodic flow analysis can be verified and used for the rest of the numerical analyses.

Ansys-Fluent software for numerical analysis of the selected pump operations are performed in the following order:

- generation of flow domain,

- generation of corresponding mesh,

- application of suitable boundary conditions,

- flow analysis and post-processing.

\subsection{The creation of flow volume of the pump}

Flow models are separately generated by using AnsysFluent software according to the design parameters. Full impeller and periodic flow volumes of the impeller are shown in Figure 1.

\subsection{Creating mesh structure and numerical analysis}

Periodic impeller model is meshed with tetrahedral cells as seen in Figure 2. Six refined layers of mesh are used for impeller volume by creating very small elements that can correctly capture the velocity and pressure gradients near walls. These refined mesh layers are also found to enhance the convergence of numerical analysis.

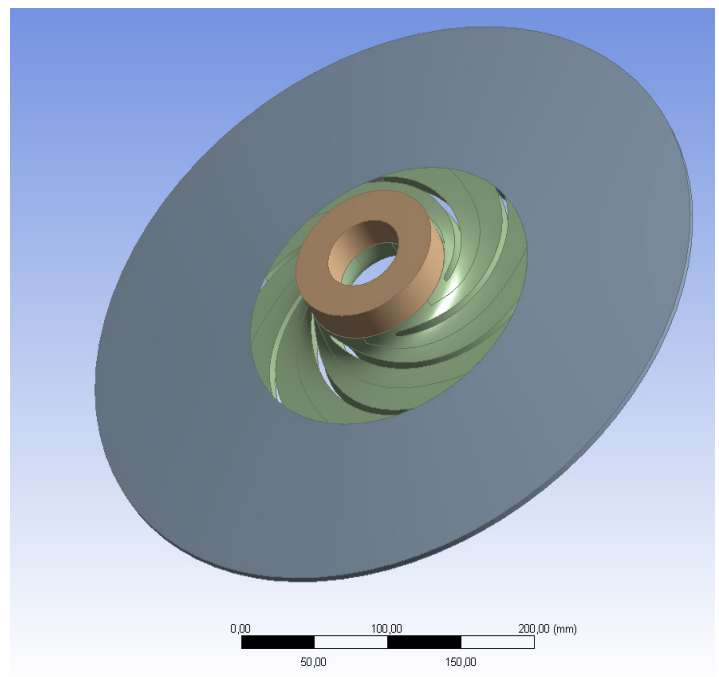

(a)

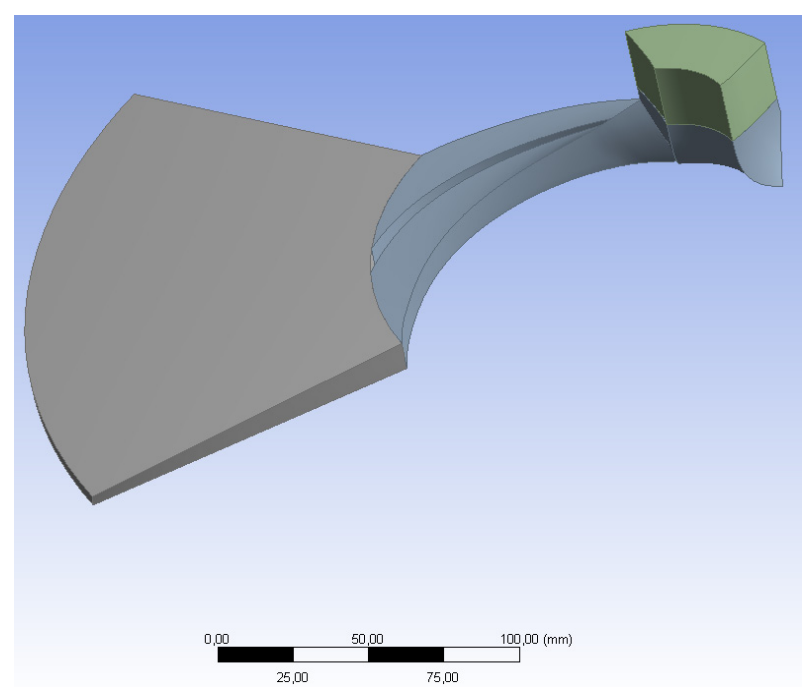

(b)

Figure 1.Full impeller (a) and periodic impeller model (b).

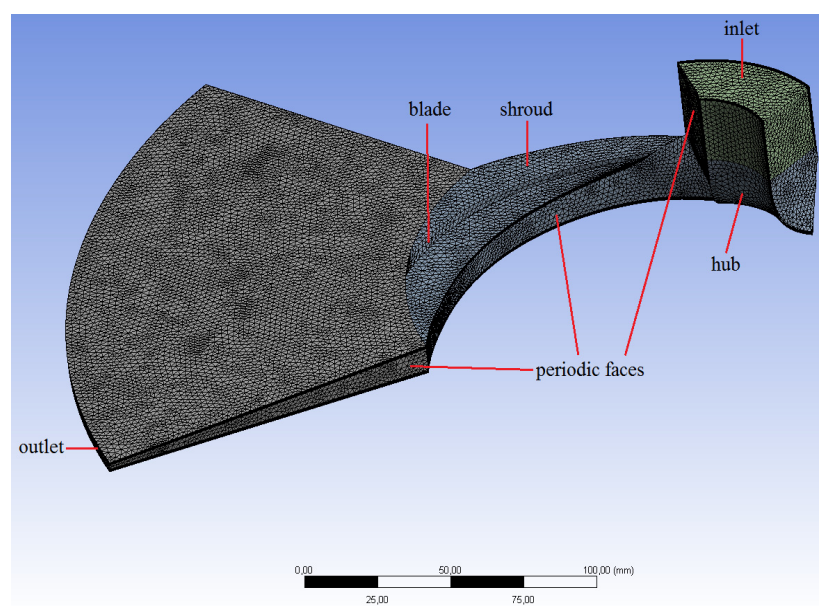

Figure 2. Mesh structure of the periodic fluid domain of impeller. 
The boundary conditions are applied corresponding to the data of the experimental study which is summarized in Table 1. Constant mass flow rate is specified on the pump inlet and fixed pressure outlet boundary is employed at the impeller outlet. Average surface roughness is assumed as $0.035 \mathrm{~mm}$ for hub, shroud and blade surfaces in accordance with the material properties. No-slip wall conditions are applied on all the walls. Maximum velocity residuals are set to $10^{-5}$. The mass flow rate and static pressure at the pump inlet and outlet are also monitored. When the overall imbalance of the four monitors is less than $0.1 \%$ or the maximum residual is reached, the analysis is assumed to be converged.

Table 1. Boundary conditions.

\begin{tabular}{|l|l|}
\hline Position & Boundary condition \\
\hline Inlet & $\begin{array}{l}\text { Mass flow inlet, 1.94 - 4.72 } \\
\mathrm{kgs}^{-1}\end{array}$ \\
\hline Inlet & Turbulent intensity, 3.5\% \\
\hline Inlet & Hydraulic diameter, 45 mm \\
\hline Outlet & Pressure outlet, 450 kPa \\
\hline Outlet & Turbulent intensity, 5\% \\
\hline Outlet & Hydraulic diameter, $8.8 \mathrm{~mm}$ \\
\hline Walls at hub and shroud & No-slip wall \\
\hline Impeller & Rotating reference frame \\
\hline Interface & General connection \\
\hline Wall function & Standard wall function \\
\hline
\end{tabular}

In the code, 3D incompressible Navier-Stokes equations are solved by using finite volume method and considering the centrifugal and Coriolis force as source terms for the flow in the impeller. The realizable $\mathrm{k}-\varepsilon$ turbulence model is preferred to take into account the turbulence behavior in the pump. For three-dimensional incompressible, unsteady flow, the continuity and momentum equations are written as follows [16-18]:

$$
\begin{gathered}
\frac{\partial}{\partial t}+\nabla(\rho \overrightarrow{\mathbf{v}})=0, \\
\frac{\partial}{\partial t}(\rho \overrightarrow{\mathbf{v}})+\vec{\nabla}(\rho \overrightarrow{\mathbf{v}} \overrightarrow{\mathbf{v}})=-\nabla P+\mu \nabla^{2} \overrightarrow{\mathbf{v}}+\rho \overrightarrow{\mathbf{g}}+\overrightarrow{\mathbf{F}} .
\end{gathered}
$$

Transport equations for the realizable $\mathrm{k}-\varepsilon$ model

$$
\begin{gathered}
\frac{\partial}{\partial t}(\rho k)+\frac{\partial}{\partial x_{i}}\left(\rho k u_{j}\right)=\frac{\partial}{\partial x_{i}}\left[\left(\mu+\frac{\mu_{t}}{\sigma_{k}}\right) \frac{\partial k}{\partial x_{j}}\right]+ \\
G_{k}+G_{b}-\rho \varepsilon-Y_{M}+S_{k}, \\
\frac{\partial}{\partial t}(\rho \varepsilon)+\frac{\partial}{\partial x_{i}}\left(\rho \varepsilon u_{j}\right)=\frac{\partial}{\partial x_{i}}\left[\left(\mu+\frac{\mu_{t}}{\sigma_{\varepsilon}}\right) \frac{\partial \varepsilon}{\partial x_{j}}\right]+ \\
\rho C_{1} S \varepsilon-\rho C_{2} \frac{\varepsilon^{2}}{k+\sqrt{v \varepsilon}}+C_{1 \varepsilon} \frac{\varepsilon}{k} C_{3 \varepsilon} G_{b}+S_{\varepsilon},
\end{gathered}
$$

where

$$
\begin{gathered}
C_{1}=\max \left[0.43 \frac{\eta}{\eta+5}\right], \\
\eta=S \times \frac{k}{\varepsilon} \\
S=\sqrt{2 S_{i j} \times S_{i j}} .
\end{gathered}
$$

Here, $G_{k \varepsilon}$ represents the generation of turbulence kinetic energy due to the mean velocity gradients, $G_{b}$ is the generation of turbulence kinetic energy due to buoyancy, $\mathrm{Y}_{\mathrm{M}}$ represents the contribution of the fluctuating dilatation in compressible turbulence to the overall dissipation rate, $C_{2}$ and $C_{l \varepsilon}$ are constants, $\sigma_{k}$ and $\sigma_{\varepsilon}$ are the turbulent Prandtl numbers for $\mathrm{k}$ and $\varepsilon$, respectively. $S_{k}$ and $S_{\varepsilon}$ are user-defined source terms. The empirical values of the model constants are taken as $C_{1 \varepsilon}=1.44, C_{2}=1.9, \sigma_{k}=1.0, \sigma_{\varepsilon}=1.2 \quad$ [18]. Solutions of the equations (3) and (4) give spatial variation of $k$ and $\varepsilon$ which, in turn, can be used to find out spatial variation of turbulent viscosity or eddy viscosity $\mu_{t}$ using the Prandtl-Kolmogorov relation [18]:

$$
\mu_{t}=\rho \times c_{\mu} \times \frac{k^{2}}{\varepsilon} .
$$

After the numerical analysis, hydraulic efficiency of the pump is calculated using equations (9-12) as stated in [19]. Here $h$ is the pump head in m unit, $\dot{Q}$ is the volume flow rate in $\mathrm{m}^{3} \mathrm{~s}^{-1}, \omega$ is the angular velocity in $\operatorname{rads}^{-1}, \dot{n}$ is the revolution in rpm and $T$ is the torque in $\mathrm{Nm}$.

$$
\begin{gathered}
P_{\text {hydraulic }}=\frac{\rho \times g \times \dot{Q} \times h}{1000}, \\
P_{\text {shaft }}=\frac{T \times \omega}{1000}, \\
\omega=\frac{\pi \times \dot{n}}{30}, \\
\eta_{\text {hydroulic }}=\frac{P_{\text {hydraulic }}}{P_{\text {shaft }}} .
\end{gathered}
$$

\section{Results and discussion}

Due to the high number of computational analysis, the authors believed that it would be impractical to conduct a full solution for the entire pump. Instead, we performed periodic simulations within the impeller passage in order to reveal the effect of blade exit angle. In order to be sure that the analysis can be performed via periodic simulations, full and periodic impeller runs are performed at the $20^{\circ}$ blade exit angle and the results are shown in figure 3 .

As seen in figures 3(a) and (b), there is only a slight difference between these two solutions. Therefore, it is suggested that the periodic configuration can be adopted in order to save computational mesh and hence the computational time for numerical analysis.

In this study, an available multi-stage centrifugal pump of the Sempa Company (a local company located in Konya, Turkey) is numerically examined. Parameters taken from the company catalogue are $\dot{Q}=72 \mathrm{~m}^{3} \mathrm{~h}^{-1}, \dot{n}=$ $2950 \mathrm{rpm}$ and $h=45 \mathrm{~m}$ for a stage. These parameters are initially used for the computational fluid dynamic analysis. After computational results, converged values are determined for each volume flow rate and different blade exit angle is shown in Figure 4. 


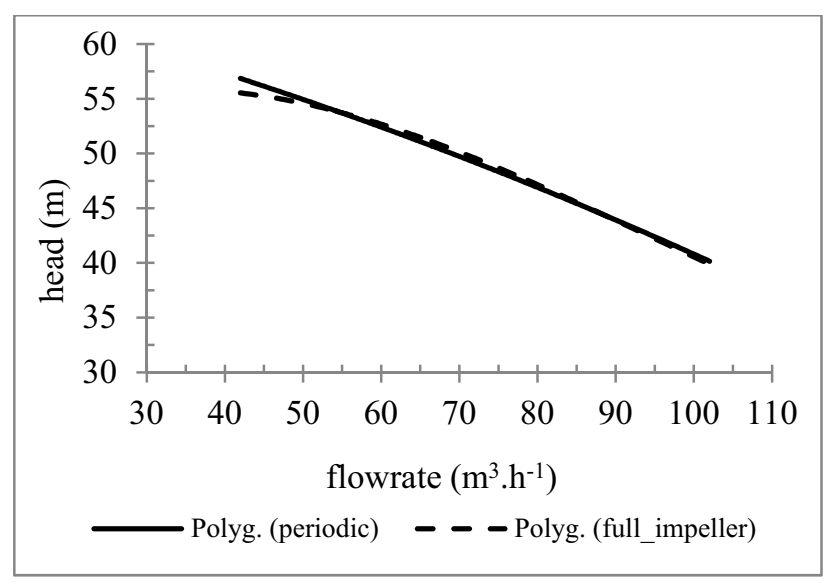

(a)

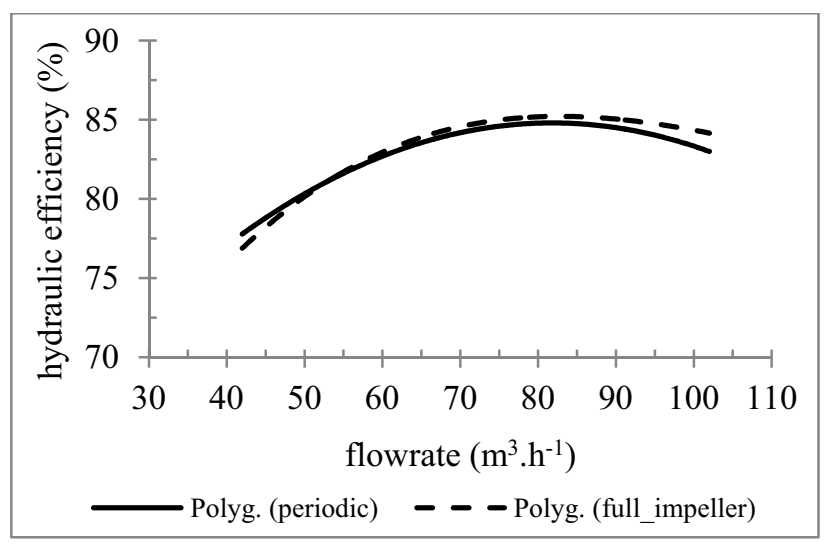

(b)

Figure 3. Variation of head (a) and hydraulic efficiency (b) with respect to the $20^{\circ}$ blade exit angle for full and passage solutions.

As seen in Figure 4(a), the head increases by increasing the blade exit angles for the flow rates higher than $82 \mathrm{~m}^{3} \mathrm{~h}^{-1}$ (over the design flow rate). As for the flow rates less than $82 \mathrm{~m}^{3} \mathrm{~h}^{-1}$, however, the head remains nearly unchanged. Figure 4(b) shows the effect of blade exit angle on the hydraulic efficiency. Here, the hydraulic efficiency increases by decreasing the exit angle of impeller blade for all flow rates.

Head, hydraulic efficiency and torque variations depending on the exit angle for design flow rate $72 \mathrm{~m}^{3} \mathrm{~h}^{-1}$ are given in Figures 5 and 6 respectively. As observed in Figure 5 , the hydraulic efficiency decreases by increasing the blade exit angle of the impeller. Head decreases by increasing the blade exit angle for the blade exit angle less than $25^{\circ}$. As for the blade exit angle is higher than $25^{\circ}$, however, the head remains nearly unchanged. The maximum hydraulic efficiency and head are found to be $84.6 \%$ and $49.2 \mathrm{~m}$ at the blade exit angle of $18^{\circ}$ respectively.

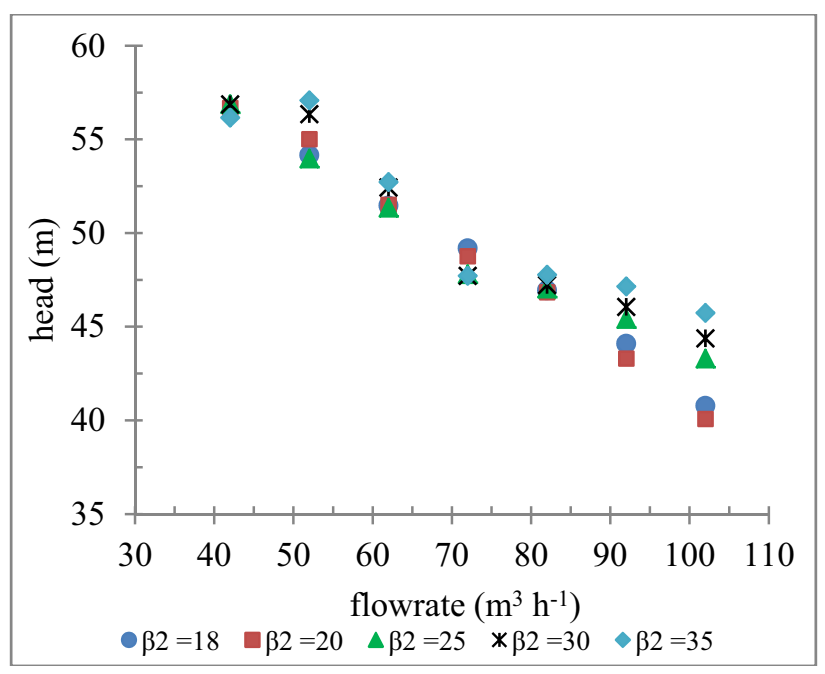

(a)

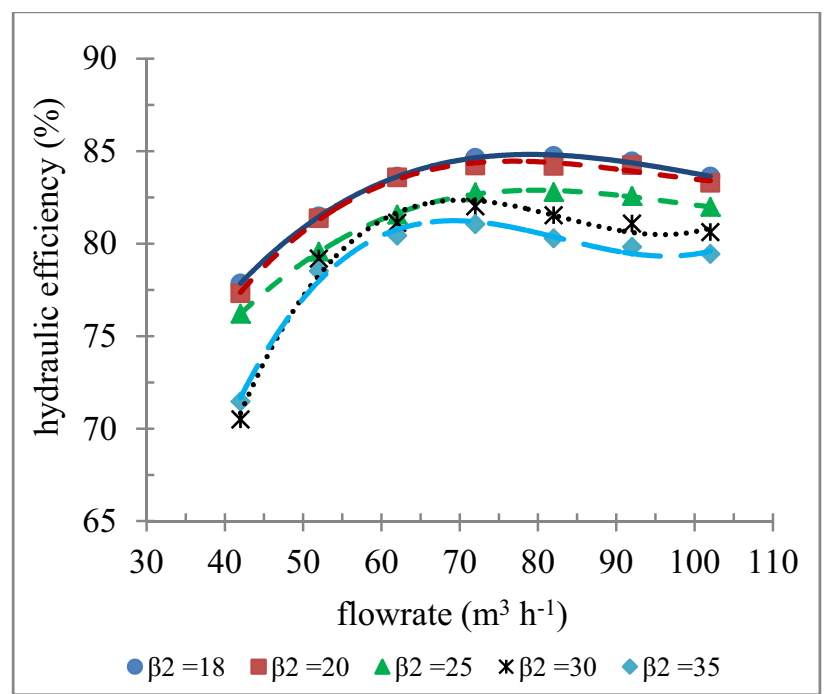

(b)

Figure 4.The effect of blade exit angles on the head (a) and hydraulic efficiency (b).

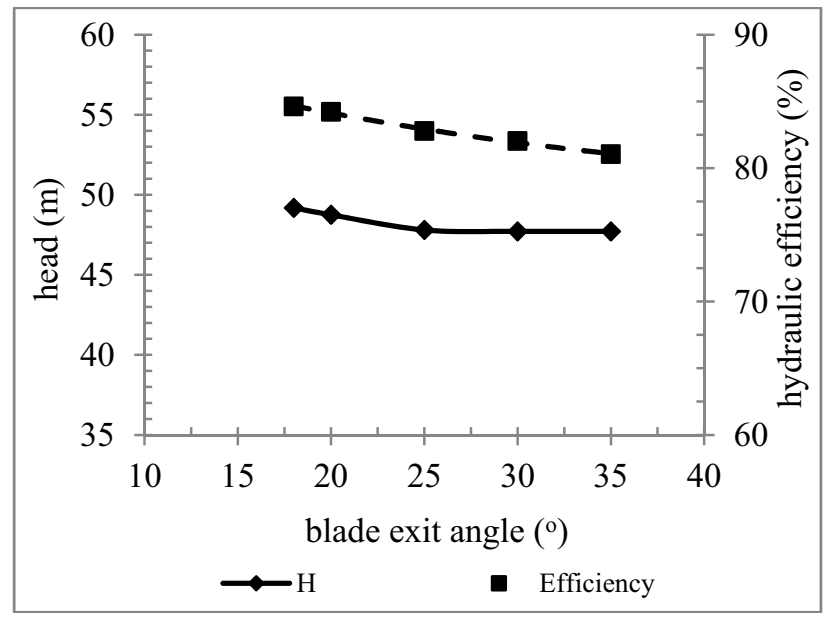

Figure 5. The effect of blade exit angle variation on head and hydraulic efficiency at the design flow rate $72 \mathrm{~m}^{3} \mathrm{~h}^{-1}$. 


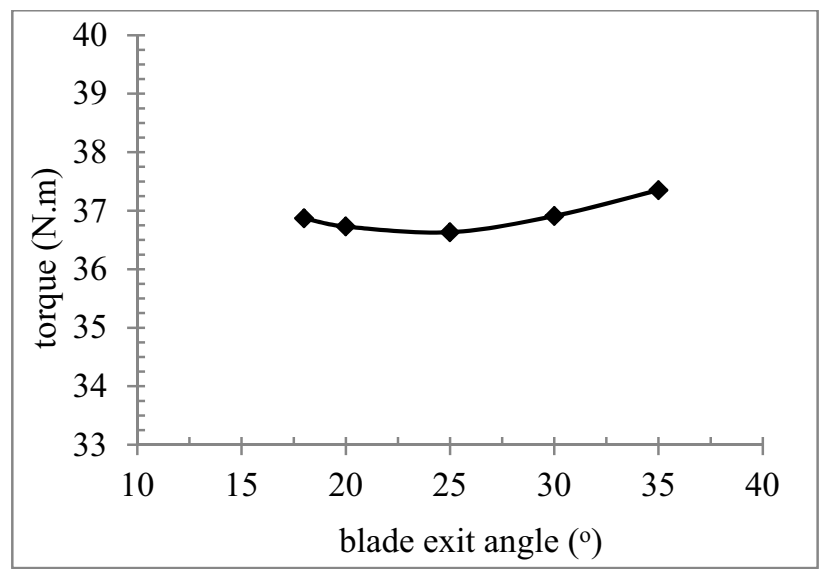

Figure 6.The effect of blade exit angle variation on torque at the design flow rate $72 \mathrm{~m}^{3} \mathrm{~h}^{-1}$.

As seen in Figure 6, torque decreases slightly between the exit angle $18^{\circ}$ and $25^{\circ}$ and increases after $25^{\circ}$. The maximum torque is calculated as $37.35 \mathrm{Nm}$ at the blade exit angle of $35^{\circ}$. The reason of this trend is attributed to the complexity of the flow structure inside the impeller and hence strength is occurred during the transfer process of the mechanical energy to the flow energy.

In order to consolidate the impact of the blade exit angles, some qualitative parameters have been studied. With this regard, total pressure and turbulent kinetic energy contours are processed and shown in Figures 7 and 8 , respectively. Streamlines are also included and shown in Figure 9 in order to demonstrate the direction of velocities within the impeller passage.

Figure 7 doesn't give detailed information about difference of pressure among the blade exit angle conditions. The distribution is generally gradual and regular. The contours look like very similar to each other. As for the turbulent kinetic energy contours, it is observed that the orientation of the blade exit angle has a significant effect on turbulent behavior. What commonly seen from sub-figures is that turbulence is generally concentrated on the blade. It is larger near the suction side than near the pressure side of the blade. High turbulence region is seen into the impeller exit passage while increasing the blade exit angle. Similar to the total pressure results, it is difficult to distinguish the difference between the streamlines. Generally, the flow keeps moving corresponding to the main velocity profile and leaves the impeller with relatively high momentum except for the region close to the suction side.

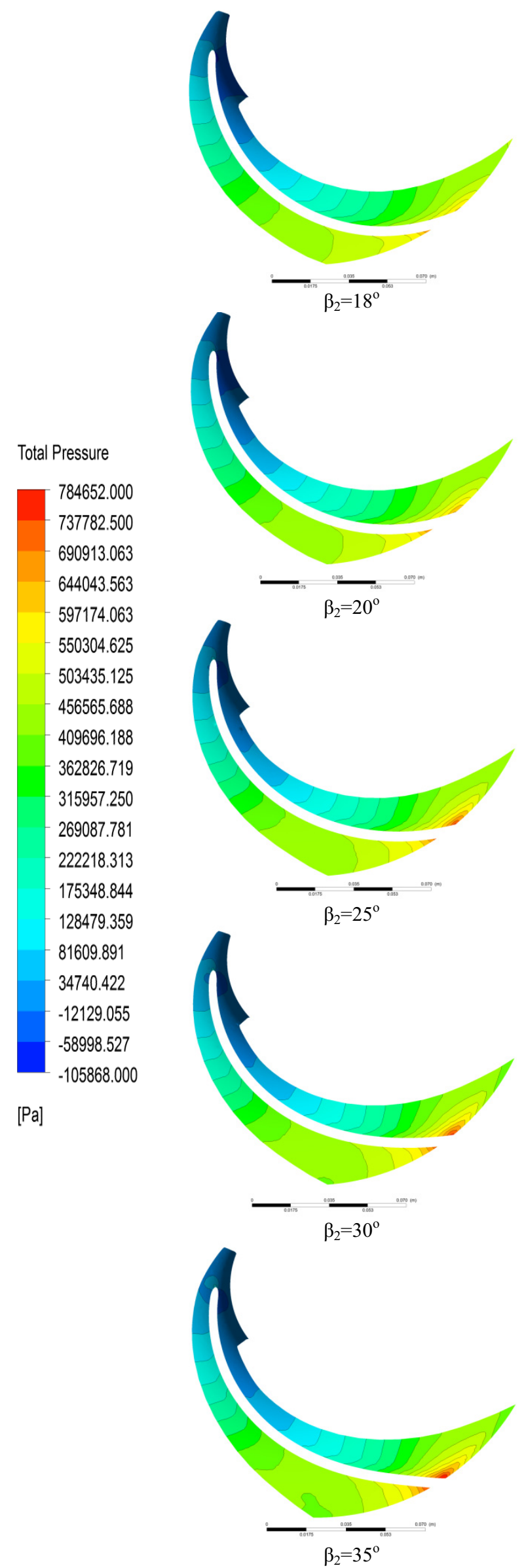

Figure 7. Total pressure variations of impeller passages for different configurations on design flow rate $72 \mathrm{~m}^{3} \mathrm{~h}^{-1}$. 


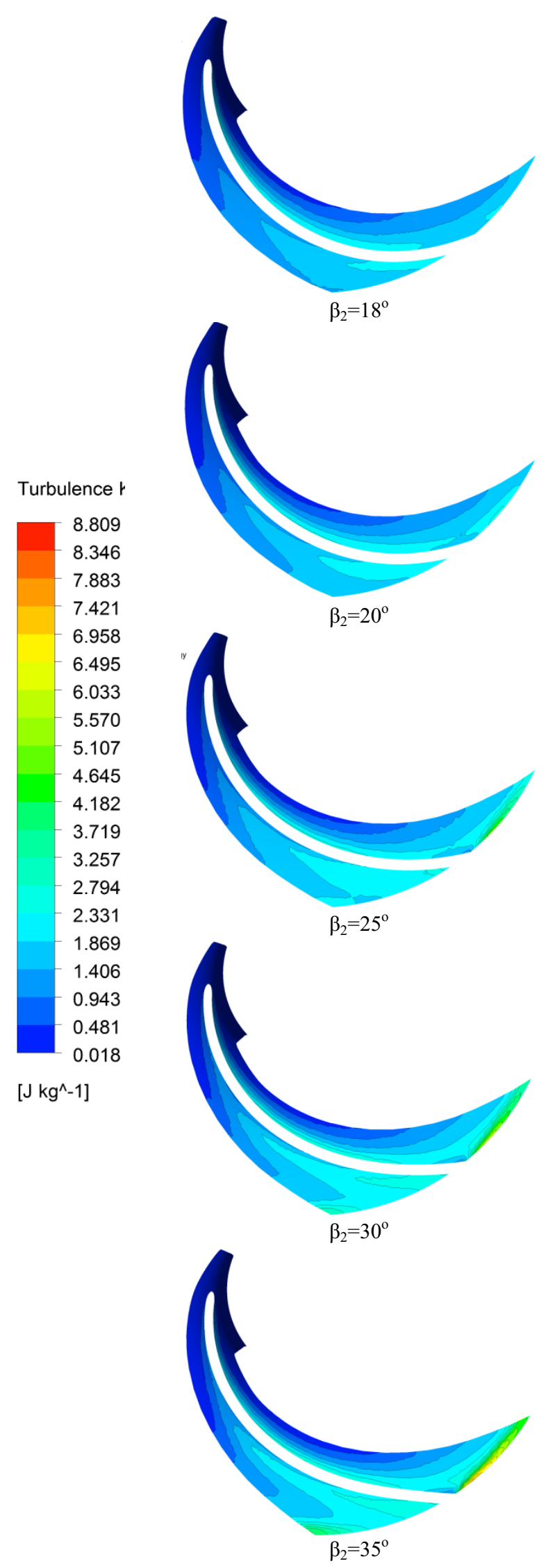

Figure 8. Turbulent kinetic energy variations of impeller passages for different configurations at the design flow rate 72 $\mathrm{m}^{3} \mathrm{~h}^{-1}$.
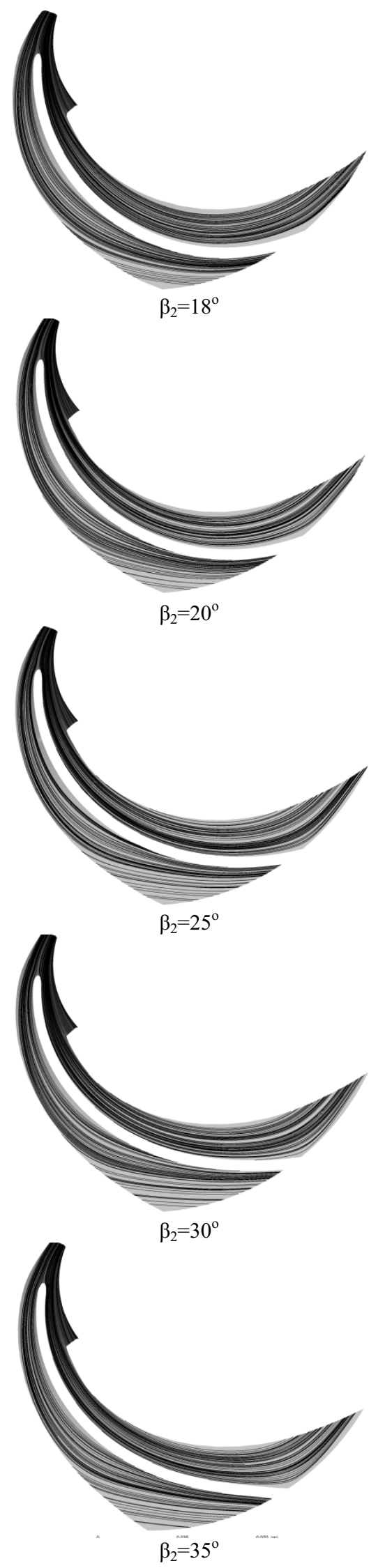

Figure 9. Streamlines of impeller passages for different configurations at design flow rate $72 \mathrm{~m}^{3} \mathrm{~h}^{-1}$. 


\section{Conclusion}

Effect of blade exit angle on the centrifugal pump impeller efficiency has been numerically investigated. How to improve the impellers performance by changing their geometric characteristics is always a challenging engineering problem. In this study, a steady state liquid flow in a centrifugal pump is investigated numerically. The continuity and Navier-Stokes equations are used accounting for the realizable $\mathrm{k}-\varepsilon$ turbulence model and the standard wall functions. Ansys-Fluent code is numerically applied to solve these equations and to perform numerical simulations, which are carried out to analyze the effect of blade exit angle on the impeller performance where all other parameters are kept constant.

A predesigned impeller geometry is obtained from several CFD analyses that blade exit angle is found to be $20^{\circ}$. Then to find an optimum value, blade exit angle of the impeller is changed in the range of $18-35^{\circ}$. Then, as demonstrated from the optimized results, it is found that the blade exit angle of $18^{\circ}$ has the maximum efficiency point at the design flow rate where hydraulic efficiency and head of the impeller are $84.6 \%$ and $49.2 \mathrm{~m}$, respectively. In future, the investigation of other effecting parameters on impeller design is planned.

Acknowledgement: This work is supported by SANTEZ program of the Ministry of Science, Industry and Technology of Turkey with number 01076.STZ.2011-2 and Selcuk University's Scientific Research Project Office. The authors also would like to thank to the partner of the project Sempa Company (http://www.sempaltd.com/en/) to their support. This study is prepared from Osman Babayigit's Ph.D. thesis.

\section{References}

1. D. Kaya, E. A. Yagmur, K.S. Yigit, F. C. Kilic, A. S. Eren, C. Celik, Energy Conversion and Management, 49 (6), 1662 (2008)
2. O. Kocaaslan, M. H. Aksoy, O. Babayigit and M. Ozgoren, Exp. Fluid Mech. 814 (2013)

3. O. Babayigit, O. Kocaaslan, M. H. Aksoy and M. Ozgoren, Exp. Fluid Mech. 807 (2013)

4. W. G. Li, J. of Appl. Fluid Mec. 4, 9, 105 (2011)

5. M. G. Patel and A. V. Doshi, Intern. J. of Emerging Tech. and Adv. Eng. 3, 1, 702 (2013)

6. M. Golcu, and Y. Pancar, World Pumps, 468, 32-40 (2005)

7. E. Korkmaz, Suleyman Demirel University Institute of Science PhD Thesis, (In Turkish) (2008)

8. C. Scheit, B. Karic and S. Becker, J. of Sound and Vibr. 331, 5, 996 (2012)

9. M. H. Aksoy, O. Babayigit, O. Kocaaslan and M. Ozgoren, Exp. Fluid Mech. 799 (2013)

10. T. Shigemitsu, J. Fukutomi and K. Kaji, Intern. J. of Fluid Mach. and Syst. 4, 3, 317 (2011)

11. Z. Yu, S. Li, W. He, W. Wang, D. Huang and Z. Zhu, HVAC\&R Research 11, 2, 263 (2005)

12. J. S. Anagnostopoulos, Computers \& Fluids 38, 2, 284-289 (2009)

13. S. D. Kyparissis, E. C. Douvi, E. E. Panagiotopoulos, D. P. Margaris and A. E. Filios, Intern. Review of Mech. Eng. 3, 3, 284 (2009)

14. Sanda B. and Daniela C. V. J. of Eng. Studies and Res. 18, 4, 32-39 (2012)

15. S.Bayraktar, C. Eralp, $4^{\text {th }}$ Congress and Exhibition on Pumps, 49 (2001)

16. Y. Liu, R. Li, K. Sun, Adv. Mater. Research, 291294, 2608-2611 (2011)

17. S.D. Kyparissis, E.C. Douvi, E.E. Panagiotopoulos, D. P. Margaris, A.E. Filios, I. Review of Mech. Eng. 3, 284 (2009)

18. Anonymous, Fluent 14.0 User Guide, Fluent Inc. (2013)

19. B. K. Baysal, Istanbul Technical University publishing house (1979) 